\title{
Vaginal Pain
}

National Cancer Institute

\section{Source}

National Cancer Institute. Vaginal Pain. NCI Thesaurus. Code C78701.

A painful sensation in the vagina. 